\title{
Determination of Positions, Velocities, and Kinetic Energies of Resonantly Excited Ions in the Quadrupole Ion Trap Mass Spectrometer by Laser Photodissociation
}

\author{
J. D. Williams and R. G. Cooks
}

Department of Chemistry, Purdue University, West Lafayette, Indiana, USA

\author{
J. E. P. Syka \\ Finnigan MAT, San Jose, California, USA
}

\author{
P. H. Hemberger and N. S. Nogar \\ CLS Division, Los Alamos National Laboratory, Los Alamos, New Mexico, USA
}

\begin{abstract}
The effects on ion motion caused by the application of a resonance AC dipole voltage to the end-cap electrodes of the quadrupole ion trap are described. An excimer laser is used to photodissociate benzoyl ions, and its triggering is phase locked to the AC voltage to follow the motion of the ion cloud as a function of the phase angle of the $\Lambda C$ signal. Resonantly excited ions maintain a coherent motion in the presence of He buffer gas, which dissipates energy from the ions via collisions. Maximum ion displacements, which depend upon the potential well depth ( $a_{z}$ value), ocrur twice each AC. cycle. Axial components of ion velocities are determined by differentiating the displacements of the distributions with respect to time. The experimental data show that these velocities are maximized when the ion cloud passes through zero axial displacement, and they compare favorably with results calculated using a simple harmonic oscillator model. Axial components of ion kinetic energies are low $(<5 \mathrm{eV})$ under the chosen experimental conditions. At low values of $q_{z}$ $(\sim 0.2)$, the width of the ion distribution increases as the ion cloud approaches the center of the trap and decreases as it approaches the end-cap electrodes. This effect is created by compaction of the ion trajectories when ion velocities are decreased. ( $/$ Am Soc Mass Spectrom 1993, 4, 792-797)
\end{abstract}

$\mathrm{R}$ esonant energy transfer is one of the oldest techniques used to manipulate ion trajectories and to detect ions in both electrodynamic (quadrupole) [1-5] and magnetic (ion cyclotron resonance) traps [6-8]. Both types of traps use resonance techriques to perform tandem mass spectrometry (MS/MS) [9-12]. Tandem experiments with the Finnigan quadrupole ion trap mass spectrometer (ITMS) utilize energy transferred from an external dipole field, derived from a supplementary $A C$ voltage applied differentially across the end-cap electrodes. The kinetic energy gained from the absorption of the applied AC signal causes ions to undergo energetic collisions with the helium bath gas and to fragment subsequently. Resonant excitation also can cause ions to increase their motion to the point that their trajectories exceed

Address reprint requests to $\mathrm{R}$. G. Cooks, Department of Chemistry, Purdue University, West Lafayette, IN 47907. the axial dimensions of the trap. This forms the basis for the resonance ejection technique, which is used to extend the mass-to-charge range of the quadrupole ion trap $[13,14]$.

Kinetic energies for stored ions are known from a variety of models and experiments to be a few $\mathrm{eV}$ or less when cooled by collisions with a buffer gas [15-19]. On the other hand, experiments and simulations have shown that ions ejected from the trap by the massselective instability scan [20] have kinetic energies that can exceed $1 \mathrm{keV}$ [21]. Other recent simulations have studied ion motion when subjected to auxiliary dipole [22-25] and quadrupole fields [26, 27] created by applying the $A C$ across the end-caps out of phase and in phase, respectively. These simulations show that ion excursions in the axial direction increase as the ion absorbs energy from the resonance AC voltage. Experimental studies on resonant excitation have focused on determining the amount of internal energy deposited 
and on the parent ion dissociation efficiency as a function of AC amplitude, frequency, and time [11, 28-30]. The kinetic energies of ions subjected to resonant excitation and simultaneously deactivated by collisions with bath gas have not been reported. Determinations of ion kinetic energies under the conditions of this dynamic equilibrium are important for a better understanding of the collision process in the ion trap and for its analytical applications, especially MS/MS experiments.

We have performed ion tomography studies using spatially resolved laser photodissociation [31, 32] of the benzoyl cation to experimentally determine, for the first time, kinetic energies of resonantly excited ions stored in the quadrupole ion trap. By firing the laser at selected phase angles of the resonant $A C$, ion motion can be tracked in small increments of time $(<1 \mu \mathrm{s})$ and the resulting displacement versus time data can be converted into ion velocities. Finally, data are presented showing how the width of the distribution varies as a function of the $A C$ phase angle at $q_{z}=0.2$. Changes in the observed distribution width are attributed to compaction of the ion trajectories as ion velocities decrease, although systematic timing errors in the laser triggering circuit may provide a minor contribution to this effect.

\section{Experimental}

The apparatus has been described previously [31]. The ion trap control electronics were modified to provide a master trigger pulse at the first zero crossing of the supplementary AC voltage. Detection of this zero crossing always occurred within \pm 140 ns of this point since the main clock for the triggering circuit operated at a frequency of $3.58 \mathrm{MHz}$ (the clock rate of the ITMS Scan Acquisition Processor). The master pulse enabled a delay generator (Stanford Research DG 535) to produce a slave pulse to control the triggering of the laser. The laser could be fired at any selected point in time (specified phase angle) during the resonant excitation period by adjusting the delay between the master and slave pulses. The laser fired $1.000 \mu \mathrm{s}$, with a jitter of 2 ns, after the slave pulse. Signals from a photodiode, which was irradiated by a portion of the laser beam, and the supplementary $\mathrm{AC}$ voltage applied to the exit end-cap electrode were sent to a $100 \mathrm{MHz}$ digital oscilloscope (Gould Model 5110) to measure the timing of the laser firing with respect to the phase angle of the $\mathrm{AC}$.

A XeCl excimer laser (Questek madel 2200) provided photons at a wavelength of $308 \mathrm{~nm}$ with a pulse width of $15 \mathrm{~ns}$ for photodissociation. Pulse energies, monitored prior to entry into the vacuum chamber, were typically $1-3 \mathrm{~mJ}$. To measure the laser beam width at the focal point, which was located inside the trap, a photodiode array was set up on an equivalent beam line external to the vacuum chamber. At the focus, the beam distribution was gaussian along the $z$ axis with a width of $0.64 \mathrm{~mm}$ [full width half maximum (FWHM)] with a fluence of $0.2-0.7 \mathrm{~J} / \mathrm{cm}^{2}$.

The spatial distribution of benzoyl ions was determined by monitoring the abundance of the photodissociation product in $\mathrm{C}_{6} \mathrm{H}_{5}^{+}(\mathrm{m} / z \mathrm{z7})$ as a function of the laser beam position. The laser beam was positioned along the $z$ axis of the trap by a mirror mounted on a translatable stage, through an axial slot cut in the ring electrode [31].

The mass spectrometer was operated using the scan editor program from the Finnigan ITMS software. The data acquisition sequence was composed of (1) electron impact ionization of acetophenone (1-2 ms), (2) isolation of the benzoyl ion $(m / z 105)$ and adjustments of the amplitude of the $1.1 \mathrm{MHz} \mathrm{RF}$ voltage applied to the ring electrode so as to store ions of $m / z 105$ at a selected value of the Mathieu parameter, $q_{z}$, (3) application of a supplementary $A C$ voltage to the end-caps at an appropriate frequency and amplitude to resonantly excite the benzoyl ions for $10 \mathrm{~ms}$, and (4)

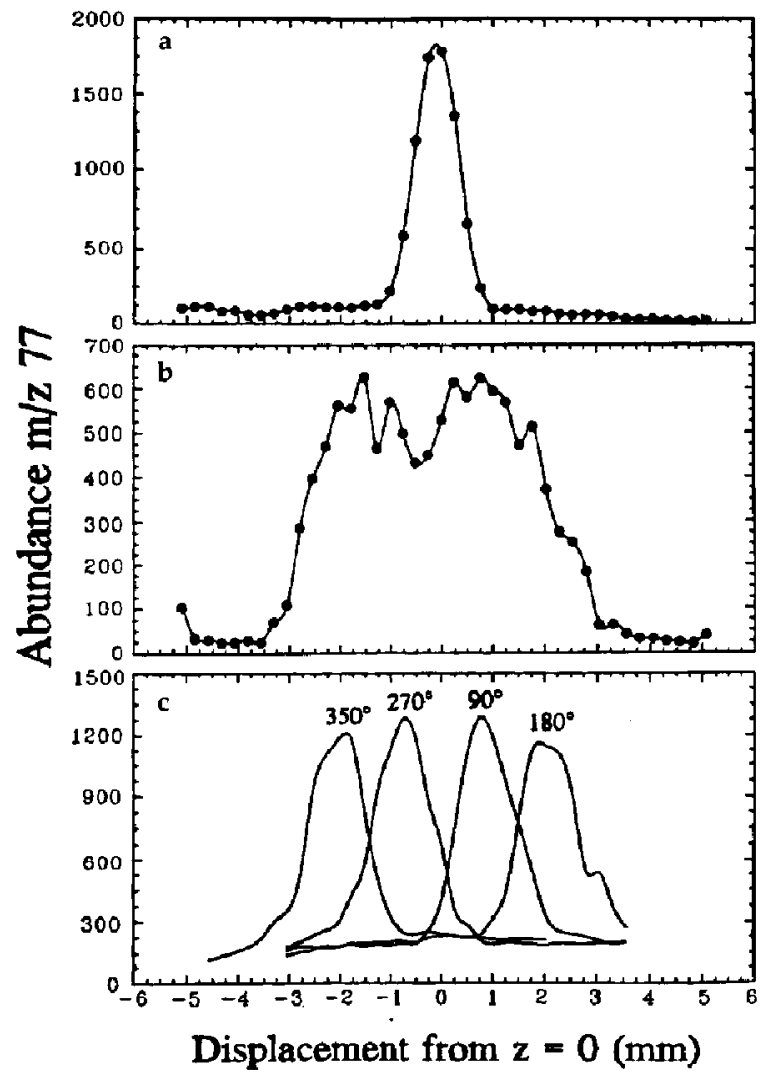

Figure 1. Distributions produced by spatially resolved photodissociation of benzoyl ions (a) prior to the application of the supplementary AC voltage, (b) without phase-locking of the laser to the supplementary $\mathrm{AC}$ voltage, and (c) with phaselocking at specified phase angles of the $\mathrm{AC}$ excitation voltage. For b and c, photodissociation was performed $7 \mathrm{~ms}$ into the resonance excitation period to obtain the ion distributions under equilibrium conditions. 
ejection and detection of product ions using the massselective instability scan [20]. The laser was triggered to fire once, approximately $7 \mathrm{~ms}$ into the excitation period. This $7 \mathrm{~ms}$ delay allowed the ions to reach their steady-state displacements [33, 34]. The amplitude of the $A C$ voltage in each experiment was adjusted to limit the number of $\mathrm{C}_{6} \mathrm{H}_{5}^{+}$ions created by collisioninduced dissociation (CID) to less than $10 \%$ of the undissociated $m / z 105$ ion current. The presence of these product ions did not affect the treatment of the data and was ignored. One hundred scans were acquired at each laser beam position and the data were averaged.

Acetophenone (Analabs) was admitted into the vacuum chamber through a variable leak valve to an indicated partial pressure of $8 \times 10^{-8}$ torr. Helium buffer gas pressures were typically $1 \times 10^{-5}$ torr. The base pressure for the instrument is $8 \times 10^{-9}$ torr. All pressures are uncorrected.

\section{Results and Discussion}

Benzoyl inns, stored at a $q_{z}$ value of 0.4 , were found by photodissociation to be present along the $z$ axis in a gaussian-shaped distribution centered at $z=0$ (Figure 1a). This result is similar to that reported previously for ions that also have been cooled to the center of the trap through collision with helium [31]. Figure $1 b$ shows the distribution of benzoyl ions as determined by photodissociation when the laser is fired $7 \mathrm{~ms}$ after a $40 \mathrm{mV}_{0-p}, 160.5 \mathrm{kHz}$ resonant $\mathrm{AC}$ signal is applied to the end-cap electrodes of the trap. As expected, ion motion is significantly altered by the application of the resonant voltage. Triggering of the laser was not phase locked to the excitation voltage for this experiment [31]. Repeating this experiment with the laser fired at phase angles of $90^{\circ}, 180^{\circ}, 270^{\circ}$, and $350^{\circ}$ with respect to the AC signal yielded distributions of photoproduct with maximum abundances displaced from $z=0$ (Figure 1c). Phase-locked triggering eliminates ambiguities in the data created by sampling over a large range of phase angles as done for the data shown in Figure 1b. More significantly, this experiment shows that the resonantly excited ions move as a function of the $\mathrm{AC}$ phase angle, and they travel as a coherent packet in the presence of $\mathrm{He}$ bath gas. An important conclusion, from this experiment, is that collisions with He buffer gas remove kinetic energy from the ions without destroying the coherence of the cloud (i.e., the buffer gas acts as a viscous drag [35]). Thus, the system can be modeled as a forced-damped oscillator [36] where the supplementary AC voltage is the forcing function and the buffer gas collisions provide damping.

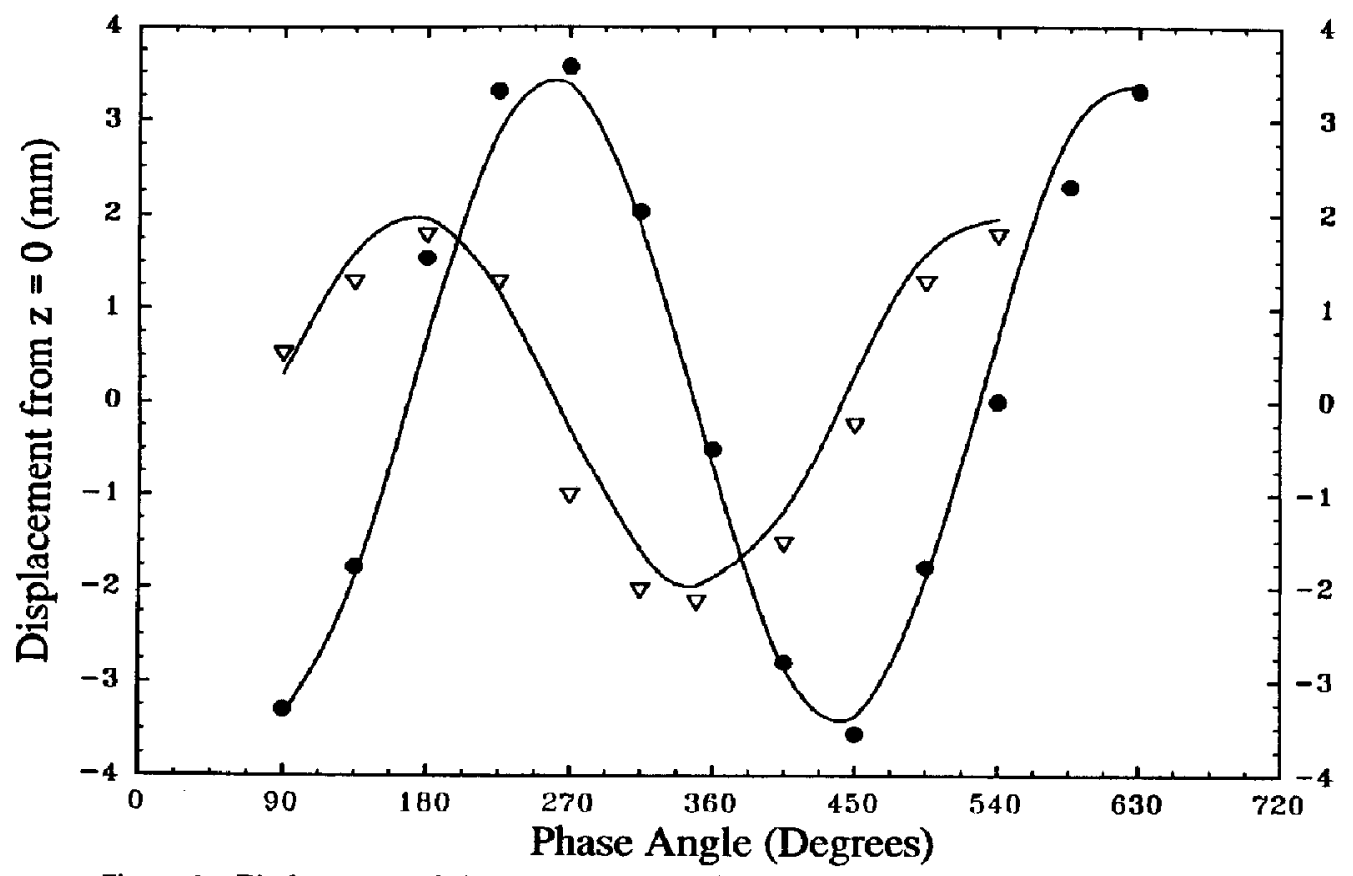

Figure 2. Displacements of the ion distributions (measured at the maxima of the individual distributions) as a function of phase angle at $q_{z}$ values of 0.2 and 0.4 . Positive displacement is for ion cloud movement toward the filament end-cap. Phase angles are relative to the $\mathrm{AC}$ signal applied to the exit end-cap. $-q_{z}=0.2$, full line is a plot $z(\mathrm{~mm})=3.48(\mathrm{~mm}) \times\left(\sin 2 \pi w_{z} t-169^{\circ}\right)$; $\nabla=q_{z}=0.4$, full line is a plot $z(\mathrm{~mm})=1.97(\mathrm{~mm}) \times\left(\sin 2 \pi w_{z} t-7^{\circ}\right)$. 
More data were taken to understand the phase dependence of the ion cloud position. The results of these experiments are summarized in Figure 2, which shows the mean displacements of the ion distributions plotted as a function of phase angle of the applied AC voltage. The supplementary AC signal had a frequency of $85.5 \mathrm{kHz}$ and an amplitude of $74 \mathrm{mV}_{0-p}$ for the data taken at $q_{z}=0.2$. (The experimental conditions for the data taken at $q_{z}=0.4$ were the same as previously stated.) The displacement of the ion cloud at $q_{z}=0.2$ is greater than at $q_{x}=0.4$, and this is attributed to the larger amplitude of the supplementary AC voltage and the shallower potential well depth $[15,37](6.5 \mathrm{eV}$ compared with $27 \mathrm{eV}$ as estimated by the Dehmelt model).

The data points can be fitted by a sinusoidal function $\left(z=z_{\text {tala }} \sin \left(2 \pi \omega_{z}+\phi\right)\right)$, where $\omega_{z}$ is the fundamental axial angular frequency of the ions, $\phi$ is the fitted phase shift, and $z_{\max }$ is the fitted maximum displacement of the distribution. This function is predicted using a simple harmonic oscillator model [15]. In a forced-damped oscillator, the output (ion motion) will approximate a harmonic oscillation of the same frequency as the input (applied $\mathrm{AC}$ ). If the input frequency, $\omega$, matches the natural resonant frequency of the system, $\omega_{z}$, the output is expected to lag the input by $90^{\circ}$. The phase shift is expected to be less than $90^{\circ}$ when $\omega<\omega_{z}$ and greater than $90^{\circ}$ for $\omega>\omega_{z}$ [36]. In this work, the ion motion is phase-shifted by $169^{\circ}$ $\left(q_{z}=0.2\right)$ and $77^{\circ}\left(q_{z}=0.4\right)$ with respect to the applied AC. These phase shifts are not unexpected, given the impedance to ion motion provided by the bath gas. The magnitude of the phase shifts varies with experimental conditions such as amplitude and frequency of the $A C$, He pressure, and trapping potential; however, systematic studies of these parameters were not performed. Finally, additional effects on the phase shifts may be due to the higher-order field components associated with the "stretched" geometry of the ion trap [38]. Various experiments have shown ion motion can be significantly altered from that produced by an "ideal" trap, especially for ions displaced from the center of the trap, by these field components [39-41].

Figure 3 shows the ion velocities, under the two sets of conditions used, obtained by differentiating the displacements (compare with Figure 2) with respect to time $(v=d z / d t)$. Figure 3 compares the experimental data to the results calculated from the fitted data [15] $\left(v=2 \pi w_{z} z_{\max } \cos \left(2 \pi w_{z}+\phi\right)\right)$. The experimental axial component velocities deviate slightly from those calculated by the simple harmonic motion model, partly because poorly fitted displacement data points (Figure 2), when differentiated, give an even poorer fit to velocities calculated by the model. Maximum ion velocities and kinetic energies are achieved when the ion cloud passes through zero axial displacement (minimum potential energy) (namely, at phase angles of $169^{\circ}$ and $349^{\circ}$ for $q_{z}=0.2$ and at phase angles of $257^{\circ}$ and $437^{\circ}$ for $q_{z}=0.4$ ). Most activating collisions

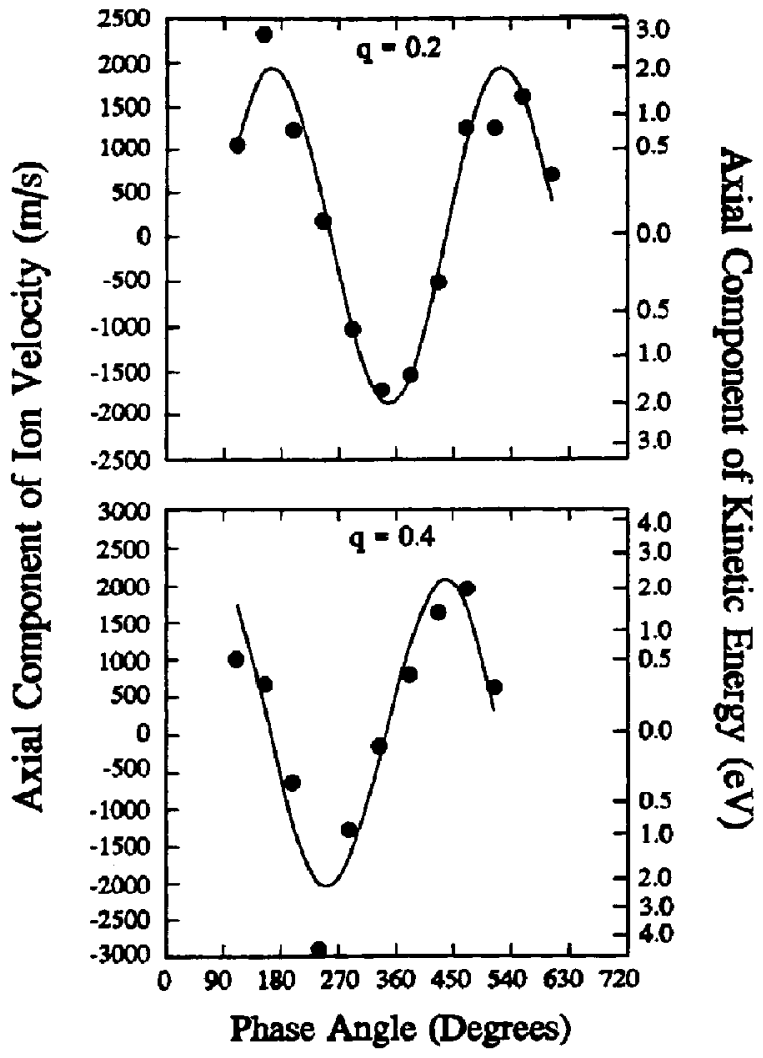

- Experimental Data - Simple Harmonic Motion

Figure 3. Axial component of ion velocities and kinetic energies calculated from the data shown in Figure 2.

with buffer gas are expected to occur in this region due to the increased ion velocities, and dissociation of the excited ions and storage of fragment ions are expected to be enhanced in this space $[28,34]$. The observed axial component of the ion kinetic energies is low ( $<5 \mathrm{eV})$ at all phase angles, which is consistent with the small amount of collision-induced fragmentation observed.

The experimental data can also be used to estimate the axial component of the kinetic energy at $A C$ voltages typical of those used in MS/MS experiments. The maximum axial ion displacements may increase from 2 $\mathrm{mm}$ (Figure 2) to perhaps $4 \mathrm{~mm}$ by increasing the amplitude of the resonant $A C$ voltage from $40 \mathrm{mV}_{0-p}$ (these experiments) to $80 \mathrm{mV}_{0-p}$ (typical MS/MS experiments) when $g_{z}=0.4$. The axial component of ion velocities will be doubled, and the axial component of ion kinetic energies will consequently increase by a factor of 4 . The maximum axial component of ion kinetic energies will be $\approx 9 \mathrm{eV}$ for an ion of $m / z 105$ under these conditions. This is consistent with the occurrence of significant CID, as is observed when the resonant excitation voltage is set at $80 \mathrm{mV}_{0-p}$. 
Under the conditions of these experiments, the width of the ion distribution, measured at the half maximum, changes as a function of phase angle. This feature is shown in Figure 4 for the data taken at $q_{z}=0.2$. The ion cloud expands as it approaches zero displacement and contracts as it approaches its maximum excursion (compare with Figure 2). The changing width of the ion distribution is attributed to velocity compaction of the ion cloud when it is displaced at $z_{\max }$, which can be attributed to the reduction of the velocity of the ion cloud when it approaches $z_{\max }$. A potential source of broadening arises from the laser firing within a $280 \mathrm{~ns}$ window of a selected phase. This broadening can be calculated using the period of the applied AC frequency $(11.7 \mu \mathrm{s})$ and the timing error in generation of the master pulse. The error in laser triggering is quite small ( $\leq 2 \mathrm{~ns}$ ) compared with the error in detection of the zero crossing. This limits the precision of the experiment in terms of phase angle to $\pm 4.29^{\circ}\left[( \pm 0.140 \mu \mathrm{s} / 11.7 \mu \mathrm{s}) \times\left(360^{\circ}\right)\right]$. This error can be converted into displacement error by using the sinusoidal fit equation (Figure 2). Measurement errors of $\pm 0.266 \mathrm{~mm}$ occur at zero crossings and $\pm 0.010 \mathrm{~mm}$ at $z_{\max }$ for each ion. Although this distorts the FWHM of the individual ion distributions, as shown in Figure 4, it does not negate the general observation of the cyclical variations in FWHM with phase angle. The trend to lower maximum FWHM values in each cycle is not understood and will be studied further. Analysis of the width of the ion distributions taken at higher values of $q_{z}(\sim 0.6)$ become complicated because the increased contributions of higher-order frequency components add to ion motion in a nonlinear fashion (i.e., these components are not multiples of $f_{z}$ ) $[2,15,42]$. Monitoring the ion cloud for many $\mathrm{AC}$ cycles to experimentally determine the frequencies of the higher-order frequency components and the extent that they contribute to ion motion would be of interest [42].

\section{Conclusions}

These experiments demonstrate the power of laser tomography for the determination of ion displacements and velocities. These data represent the first direct measurement of displacements and velocities for resonantly excited ions in the quadrupole ion trap. Measured and estimated kinetic energies provide experimental support for the hypothesis that trapped ions have low kinetic energies. Further experiments are in progress to better understand phase shifts between the resonant excitation voltage and ion motion, as are experiments to understand the origins of chemical-dependent mass shifts that have been observed for ion traps of "ideal" [38] and "stretched" $[43,44]$ geometry.

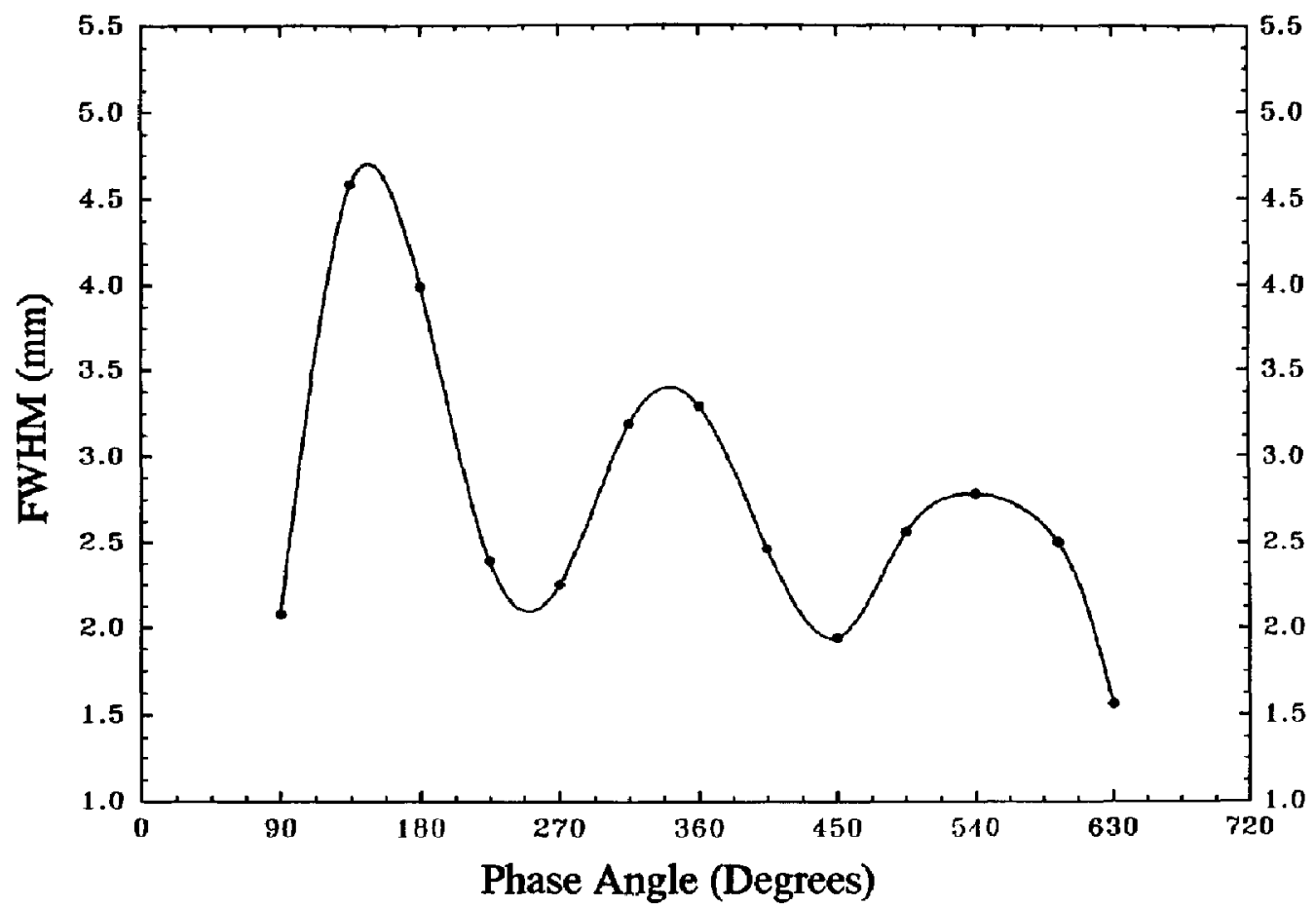

Figure 4. Width (FWHM) of the ion distribution plotted as a function of phase angle for $q_{z}$ value of 0.2 


\section{Acknowledgments}

This work was supported by the Graduate Research Assistant program at Los Alamos National Laboratory and the National Science Foundation (CHE 87-21768). We thank Mary Cisper for writing the data acquisition sequence, and Mike Alexander for his valuable comments and suggestions.

\section{References}

1. Fischer, E. Z. Phys. 1959, 156, 1.

2. Wuerker, R. F.; Shelton, H.; Langmuir, R. V. J. Appl. Phys. $1959,30,342$.

3. Rettinghaus, V. von G. Z. Agnew. Phys. 1967, 22, 321.

4. Fulford, J. E.; Hoa, D.-N.; Hughes, R. J.; March, R. E.; Bonner, R. F, Wong, G. J. J. Vac. Sci. Technol. 1980, 17, 829

5. Syka, J. E. P.; Fies, W. J. Jr. Proceedings of the 35th ASMS Conference on Mass Spectrontetry and Allied Topics; Denver, CO, May 24-29, 1987; $p 767$.

6. Wobschall, D.; Graham, J. R.; Malone, D. P. Phys. Rev. 1963, $131,1565$.

7. Baldeschwieler, J. D. Science 1968, 159, 263.

8. Comisaruw, M. B.; Marshall, A. G. Chem. Phys. Lett. 1974, 25, 282.

9. Kaplan, F. J. Am. Chem. Soc. 1968, 90, 4486.

10. Cody, R. B.; Burnier, R. C.; Freiser, B. S. Anal. Chem. 1982, 54, 96.

11. Kelley, P. E.; Stafford, G. C. Jr.; Syka, J. E. P.; Reynolds, W. E.; Todd, J. F. J. Adv. Mass Spectrom. 1985, 10, 869.

12. Louris, J. N.; Cooks, R. G.; Syka, J. E. P.; Kelley, P. E.; Stafford, G. C. Jr.; Todd, J. F. J. Anal. Chem. 1987, 59, 1677.

13. Kaiser, R. E. Jr.; Louris, J. N.; Amy, J. W.; Cooks, R. G. Rapid Contmun. Mass Spectrom. 1989, 3, 225.

14. Kaiser, R. E. Jr.; Cooks, R. G.; Stafford, G. C. Jr.; Syka, J. E. P.; Hemberger $_{f}$ P. H. Int. J. Mass Spectrom. Ion Processes 1991, 106,79 .

15. March, R. E.; Hughes, R. J. Quadrupole Starage Mass Spectrometry; Wiley: New York, 1991, and references within.

16. Schaal, II.; Schmeling, U.; Werth, G. Appl. Phys. 1981, 25, 249.

17. Siemers, I; Blatt, R.; Sauter, T.; Neuhauser, W. Phys. Rev. A $1988,38,5121$.

18. Schubert, M.; Siemers, 1,; Blatt. R. Appl. Phys. B 1990, 51, 414.

19. Vedel, F; Vedel, M. I. Mod. Opt. 1992, 39, 431.

20. Stafford, G. C.; Kelley, P. E.; Syka, J. E. P.; Reynolds, W. E.; Todd, J. F. J. Int. J. Mass Spectrom. Ion Processes 1984, 60. 85.

21. Reiser, H.-P; Kaiser, R. E. Jr.; Savickas, P. J.; Cooks, R. G. Int. J. Mass Spectrom. Ion Processes 1991, 106, 237.

22. Pranzen, J. Int. J. Mass Spectrom. Iom Processes 1991, 106, 63.
23. March, R. E.; Londry, F. A.; Alfred, R. L.; Todd, J. F. J.; Penman, A. D.; Vedel, F.; Vedel, M. Int. I. Mass Spectrom. Ion Processes 1992, 110, 159.

24. Julian, R. K. Jr.; Reiser, H.-P.; Cooks, R. G. Int. J. Mass Spectrom. Ion Processes, 1993, 123, 85.

25. Reiser, H.-I'; Julian, R. K. Jr,; Cooks, R. G. Int. I. Mass Spectrom. Ion Processes 1992, 121, 49.

26. March, R. E.; McMahon, A. W.; Todd, J. F. J.; Vedel, F. Int. I. Mass Spectrom. Ion Processes 1989, 95, 119

27. March, R. E; McMahon, A. W.; Allinson, E. I.; Londry, F. A.; Alfred, R. L.; Todd, J. F. J.; Vedel, F. Int. J. Mass Spectrom. Ion Processes 1990, 109, 99.

28. Johnson, J. V.; Yost, R. A.; Kelley, P. E.; Bradford, D. C. Anal, Chem. 1990, 62, 2162.

29. Gronowska, J.; Paradisi, C.; Traldi, P.; Vettori, U. Rapid Commun. Mass Spectrom. 1990, 4, 306.

30. Charles, M. J.; McLuckey, S. A.; Glish, G. L. Proceedings of the 40th ASMS Conference on Mass Spectrowetry and Allied Topics, Washington, DC, May 31-June 5, 1992; p 57.

31. Hemberger, P. H.; Nogar, N. S.; Williams, J. D.; Cooks, R. G.; Syka, J. E. P. Chem. Phys. Lett. 1992, 191, 405.

32. Hemberger, P. H.; Alexander, M. L.; Cisper, M. E.; Nogar, N. S.; Williams, J. D.; Cooks, R. G.; Syka, J. E. P. Proceedings of the 40th ASMS Conference on Mass Spectrometry and Allied Topics, Washington, DC, May 31-June 5, 1992; p 521.

33. Alexander, M. L.; Hemberger, P. H.; Cisper, M. E.; Nogar, N. S.; Williams, J. D.; Cooks, R. G.; Syka, J. E. I'. Presented at FACSS XIX; Philadelphia, PA, September 20-25, 1992.

34. Hemberger, P. H.; Alexander, M. L.; Cisper, M. E.; Nogar, N S.; Williams, J. D.; Cooks, R. G.; Syka, J. E. P. to be submitted.

35. Whetten, N. R. J. Vac. Sci. Technol. 1974, 11, 515.

36. Kreyszig, E. Advanced Engineering Mathematics, 6th ed.; Wiley: New York, 1988; p 129.

37. Major, F. G.; Dehmelt, H. G. Phys. Rev. 1968, 179, 91.

38. Louris, I; Schwartz, J.; Stafford, G.; Syka, J.; Taylor, D. Proceedings of the 40th ASMS Conference on Mass Spectrometry and Allied Topics, Washington, DC, May 31-June 5, 1992; $\mathrm{p}$ 1003.

39. Williams, J. D.; Reiser, H.-P.; Kaiser, R. E. Jr.; Cooks, R. G. Int. I. Mass Spectrom. Ion Processes 1991, 108, 199.

40. Guidugli, F.; Traldi, P. Rapid Commun. Mass Spectrom. 1991, $5,343$.

41. Eades, D. M.; Yost, R. A. Rapid Commun. Mass Spectrom. 1992, 6, 573 .

42. Lammert, S. A. Ph.D. thesis, Purdue University, 1992; Chapter 5.

43. Traldi, P; Curcuto, Q; Bortolini, O. Rapid Commun. Mass Spectrom. 1992, 6, 410.

44. Traldi, P.; Faveretto, D. Rapid Commun. Mass Spectrom. 1992, $6,543$. 